\title{
Design and Fabrication of Faceted Mirror Arrays for Light Field Capture
}

\author{
M. Fuchs ${ }^{1}$, M. Kächele ${ }^{1}$, and S. Rusinkiewicz ${ }^{2}$ \\ ${ }^{1}$ University of Stuttgart, Germany \\ ${ }^{2}$ Princeton University, United States of America
}

\begin{abstract}
The high resolution of digital cameras has made single-shot, single-sensor acquisition of light fields feasible, though considerable design effort is still necessary in order to construct the necessary collection of optical elements for particular acquisition scenarios. This article explores a pipeline for designing, fabricating, and utilizing faceted mirror arrays which simplifies this task. The foundation of the pipeline is an interactive tool that automatically optimizes for mirror designs while exposing to the user a set of intuitive parameters for light field quality and manufacturing constraints. We investigate two manufacturing processes for automatic fabrication of the resulting designs: one is based on CNC milling, polishing, and plating of one solid work piece, while the other involves assembly of precision cut mirror facets. We demonstrate results for refocusing in a macro photography scenario.
\end{abstract}

Categories and Subject Descriptors (according to ACM CCS): Image Processing and Computer Vision [I.4.1]: Digitization and Image Capture-Imaging geometry

\section{Introduction}

There is an inherent tension in the design of digital cameras: on the one hand, they must enable spontaneously capturing the moment ("point-and-shoot"); on the other, they are to deliver beautiful pictures that result from a painstaking choice of photographic parameters (such as viewpoint, focus, and aperture). Manufacturers have tried to give non-experts the ability to obtain high quality by providing automatic assistants (most prominently auto-focus lenses and auto-exposure metering). These, however, have evolved into a plethora of disparate programs. For instance, the Pentax Optio I-10, a recent consumer camera, provides around 30 distinct scene modes, including presets for fireworks, pets, food, kids, etc. and software options for automatically focusing on human faces and detecting expressions such as smiles or blinks. The user is once again faced with having to make so many choices before a picture is taken that spontaneity and great photography seem ultimately incompatible.

Going beyond quality and usability, there is one design parameter in which camera manufacturers have enjoyed unquestioned success: resolution. There is, however, a profound and increasing discrepancy between typical display and capture resolution: modern digital SLR cameras record more than ten times as many pixels as an HDTV display can show. This suggests that, before it is displayed, most recorded footage is either cropped or rescaled, making no use of the available resolution.

The excess sensor resolution may be better used to facilitate post-hoc-photography: shoot now, choose parameters later. Doing this requires collecting many rays from
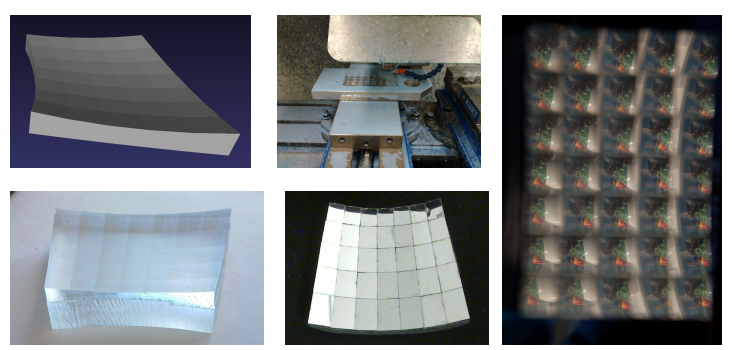

Figure 1: Design and fabrication pipeline. After computing an optimal shape, we use CNC machining to form a piece of acrylic into a support structure for a set of precision-cut polished stainless steel segments. This mirror may be used for single-shot capture of a light field with 35 views. 
the scene — a light field [LH96] — and previous systems have used modified cameras with lenslet arrays to capture sufficient data for refocusing and small viewpoint change $\left(\left[\mathrm{NLB}^{*} 05, \mathrm{GZC}^{*} 06\right]\right)$.

We investigate an alternate approach based on combining high-resolution cameras with segmented mirrors (Figure 1). These systems can be used in conjunction with conventional digital cameras, do not require modifying camera internals (such as by breaking apart the camera body or cutting open a lens), are not heavier or more sensitive to transport than commodity DSLR lenses, and can be purpose-built for roughly the same cost as quality lenses (under USD 1000).

This paper investigates a pipeline for designing, manufacturing, and utilizing such mirror arrays for catadioptric light field capture. Though we restrict the design space for our faceted mirrors, there remains a bewildering set of design choices. Moreover, most of the design parameters are not directly related to the user's goals, which are phrased in terms of how practical the mirror will be to manufacture and the resolution, coverage, and quality of the resulting light field. Therefore, the heart of our pipeline is an interactive tool (Section 3): the user is free to specify constraints and explore tradeoffs phrased in terms of intuitive quantities, while the program automatically optimizes for mirror parameters and provides feedback on manufacturing cost. We explore several case studies of mirrors designed using our program, with very different angular coverage, working volume, and intended imager (Section 4 ).

Once a mirror has been designed, it must be fabricated, and we have investigated two different manufacturing technologies (Section 5). The first approach is to CNC-mill a complete multi-facet mirror surface out of a single block of brass, then polish and plate it. The second approach is to cut discrete mirror facets and assemble them onto a support structure CNC-milled from plastic. We will discuss the challenges these methods pose and their respective advantages.

\section{Basics and Related Work}

Light fields (introduced to graphics by Levoy and Hanrahan [LH96], and, under the name "lumigraph," by Gortler et al. [GGSC96]) exhaustively model the radiance emitted by a static scene, and, outside participating media, allow computing images for any describable camera. Thus, they enable photographic applications such as refocusing, viewpoint change, and synthetic aperture modification. As a data structure, a light field maps a ray in space identified by four spatial coordinates to the radiance transported along that ray.

The task of light field acquisition is to sample the 4-D space of rays, usually along 2-D slices. Initial approaches assumed a static scene, and therefore could record it with a moving camera. This could either be hand-held [GGSC96] or moved using an automated gantry [LH96] or motorized translation stage [UWH*03,IMG00]. Later techniques speed up the acquisition, often enabling capture of dynamic scenes:

Camera arrays combine the simultaneous exposures of dozens of cameras to acquire the light field [YEBM02, $\left.\mathrm{WJV}^{*} 05\right]$. This enables video capability, and can be used for feeding a 3D display in real time [MP04], but requires a large and costly setup.

Lens arrays rely on optical components to slice the light field. Yang [Yan00] arranged 88 lenses in front of a flatbed scanner in an inexpensive setup for static scenes. Other designs require only a single, static $2-\mathrm{D}$ sensor and allow near instantaneous capture: $\mathrm{Ng}$ et al. [NLB $\left.{ }^{*} 05, \mathrm{Ng} 06\right]$ added a micro-lens array on the sensor of a digital SLR camera, thus splitting the rays going through the camera aperture into subpixel sets according to their direction, enabling a compact, transportable, stable setup, but it requires camera internals to be modified and limits the application: no larger aperture can be simulated, and the viewpoint remains largely unchanged, as all captured rays have come through the camera lens aperture. Georgiev et al. [GZC $\left.{ }^{*} 06\right]$ explored a lens array embedded into the main lens, improving on the usable sensor area. Levin et al. [LHG $\left.{ }^{*} 09\right]$ presented a 4-D frequency analysis of a multi-lens-based setup optimized for refocusing. Cossairt et al. [CNR08] used a lens array at a considerable distance from the main camera, outside its body for light field transfer. This enables a wide sampling range; however, since the lens array has reflective surfaces facing the camera, either the light from this side must be blocked (enlarging the camera body), or the lens must be employed in a carefullychosen (indoor) setup that eliminates stray reflections.

Masks in the optical path close to the camera sensor permit reconstruction of full-resolution pictures for certain scene types, as demonstrated by Veeraraghavan et al. [VRA*07, $\mathrm{VAR}^{*} 08$ ]. An additional mask close to the camera primary lens adds trade-offs on time vs. spatial and angular resolution [AVR10]. Wetzstein et al. [WIH10] showed a highdynamic-range application for a modulating camera, while Ihrke et al. [IWH10] investigated the interaction between spatial and Fourier multiplexed patterns.

Mirrors use reflections off surfaces to slice the light field. Thus, in contrast to designs based around refractive elements, they do not need to avoid these reflections on the camera-visible surfaces, do not require the optical path to be encased in large, light-tight camera bodies, and place no restrictions on scene illumination. Analogously to multiple, spatially arranged lenses, designs with multiple mirrors enable instantaneous capture, as demonstrated with an array of planar mirrors by Levoy et al. [LCV*04]. Curved mirrors increase the field of view that can be observed; they can also be arranged in a plane and still record a dense light field, which makes the construction easier. Unger et al. [UWH* 03 ] used a grid of $12 \times 12$ mirror balls for light field capture, while Lanman et al. [LCW*06] used sections of 31 spherical mirrors. Especially for full spheres, a wide field of view can be 
achieved, as has been shown by Taguchi et al. with an array of mirrors $\left[\mathrm{TAV}^{*} 10\right]$ and with a single mirror and a moving camera [TARV10]. Outside light field acquisition, curved mirrors have been used to great effect to observe a single surface point (or small patch) under many directions at once, making use of an ellipsoid / spherical [War92, MSY07] or paraboloid [Dan01] geometry. Ghosh et al. [GHAO10] used the same mirror setup also for illumination, while Peers et al. [PHD06] used a rough mirror for relighting. Mukaigawa et al. $\left[\mathrm{MTK}^{*} 10\right]$ designed a polyhedral mirror based on the geometric properties of ellipsoids for a design that allows the placement of objects at one focal point and a camera at the other to perform hemispherical confocal imaging.

This work strives to construct a setup that functions as an external augmentation to existing digital cameras, providing single-exposure recoding capability. It should be compact and inexpensive, and, once manufactured, remain stable without additional fine-tuning. We wish to allow designs optimized for a variety of rendering applications, ranging from refocusing to large synthetic apertures and modest changes in viewpoint. This leads us to choose a mirror-based construction built with rapid manufacturing tools.

With regards to geometry, we choose a piece-wise planar design, as it is does not introduce distortions while allowing effective recording of small working volumes. In contrast to $\left[\mathrm{LCV}^{*} 04\right]$, we aim for a continuous surface, as this avoids gaps and occlusions. In contrast to Mukaigawa et al. [MTK $\left.{ }^{*} 10\right]$, we provide a user guided optimization pipeline for the derivation of the geometric configuration.

\section{Designing optimal mirror geometry}

\subsection{Choosing a family of mirrors}

Let us consider two possible extremes for the shape of a mirror surface. A planar mirror yields a single virtual viewpoint with field of view identical to the camera's. In contrast, a mirror in the shape of an ellipsoid of revolution, as used by Ward et al. [War92] for BRDF measurement, enables observing a fixed point from a large, continuous range of directions. Its shape is uniquely defined by its focal points - the camera position in $A$, the scene center in $B$ (see Figure 2) and the sum of distances from its surface to $A$ and $B$.

We can combine the above ideas into a segmented ellipsoidal mirror composed of planar facets: it observes a scene from many viewpoints, each of which contains a conventional virtual camera. For any ellipsoidal basis shape, we can define the facets by intersecting one (central) ray per segment from the camera against the ellipsoid, and intersecting the planes that contain the intersection points and are tangent to the ellipsoid against their neighbors. By distributing the intersection points of the viewing rays equally on the image sensor, we can make sure that roughly the same number of pixels per segment contributes to the light field.

In determining the parameters of the ellipsoid basis shape,

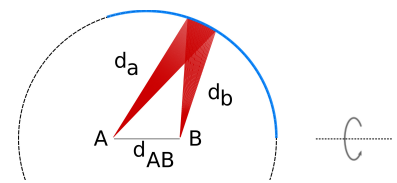

Figure 2: Consider a camera in point $A$ and a scene point $B$. The mirror surface that reflects rays from each camera direction into $B$ is the one that keeps the length of each ray pair $d_{A}+d_{B}$ constant. It is a subsection of an ellipsoid of revolution uniquely defined by these variables. Not considering global translation and rotation, the shape depends only on the distance $d_{A B}$ between $A$ and $B$ and the sum $d_{A}+d_{B}$.

it is sufficient to model its behavior in a 2-D cross-section that contains both points $A$ and $B$, as rotational ellipsoids are symmetric with respect to the axis $\overline{A B}$. We align $\overline{A B}$ with the user's choice of either of the image dimensions.

Thus, the parameters to be chosen for the mirror are the sum of distances $d_{A}+d_{B}$, the size of segments (both inplane along the ellipse and around the axis of revolution), and for the camera, we may choose the focal length and the orientation (i.e., which part of the ellipse it observes). Finally, we need to select the distance between scene and camera.

\subsection{Mirror design goals}

These parameters interact in complex ways, and one of our key observations is that there is not an immediate connection between them and a designer's high-level goals for light field capture. In particular, a user might think in terms of the following desiderata:

- Resolution of the acquired light field: the number of views (that is, the number of segments), and the number of sensor pixels per view.

- Coverage of the light field: the angular coverage / spread of observed viewpoints, and the maximum scene size that can be observed with a given mirror/camera combination.

- Efficiency of the acquisition: the directional uniformity of views, and the relative area of the sensor used. (The sensor is rectangular, but the mirror's projection into the camera view is not: the better the mirror fills the available sensor space, the more rays can be observed. We can also trade off efficiency for completeness of coverage by permitting the outer segments to partially exceed the sensor.)

In addition, the mirror shape cannot be chosen arbitrarily, but is subject to two types of limitations:

- Manufacturing costs: the cost of mirror and support material increases with volume; as does the cost of manufacturing (a CNC mill, for instance, needs to run longer if it is to ablate more material) and may further increase with surface area (when electroplating, the surface needs polishing after cutting with a dense tool path). 
- Setup constraints: the physical size of equipment dictates a minimum and maximum distance between the scene and the camera, while the availability of objective lenses places limits on focal length.

In order to design an mirror array satisfying the user's goals and constraints, we turn to optimization. We express the desirables and limitations in a single (non-linear) objective function (Figure 3), and use the levmar [Lou04] optimizer to find the best-compromise mirror parameters.

$$
\begin{aligned}
\mathcal{E}= & T_{\text {surface area }}^{\max }+T_{\text {bounding box width }}^{\max } \\
& +T_{\text {bound. box height }}^{\max }+T_{\text {bound. box depth }}^{\max }+T_{\text {rel. sensor coverage }} \\
& +T_{\text {scene radius }}+T_{\text {diagonal angle spread }} \\
& +T_{\text {angle uniformity }}+T_{\text {focal length }}^{\max }+T_{\text {focal length }}^{\min }
\end{aligned}
$$

where

$$
\begin{aligned}
T_{x} & :=\lambda_{x}^{2} \cdot\left(x_{\text {desired }}-x_{\text {actual }}\right)^{2} \\
T_{x}^{\max } & :=\lambda_{x_{\max }}^{2} \cdot \max \left\{0,\left(x_{\text {actual }}-x_{\text {desired }_{\text {max }}}\right)\right\}^{2} \\
T_{x}^{\min } & :=\lambda_{x_{\min }}^{2} \cdot \max \left\{0,\left(x_{\text {desired }_{\min }}-x_{\text {actual }}\right)\right\}^{2}
\end{aligned}
$$

Figure 3: Objective function for the nonlinear optimization. The individual objectives are weighted by user-controlled parameters $\lambda_{\bullet}$. For the objectives with the $T_{\bullet}$ terms, any deviation from the desired value incurs a penalty; for the others, only a deviation beyond $\left(T_{\bullet}^{\max }\right)$ or below $\left(T_{\bullet}^{\min }\right)$ the limit increases the cost function.

\subsection{Interactive mirror design}

The terms of the objective function are individually weighted with user-chosen parameters $\lambda_{\bullet}$, initialized to 1 . As there is no intrinsic best way of defining the trade-off between the parameters, we leave the choice to the user, providing instant feedback in an interactive application to support exploration of the solution space (Figure 4 and the supplementary video):

A user defines the desired properties and weights and promptly observes the actually achieved statistics of the current solution. He/she can also define the variables that are not subject to the optimization: number of views/segments and sensor dimensions. The user interface colors objectives which either have been exactly met ( $\mathrm{min} / \mathrm{max}$ limits) or are within $1 \%$ of the desired value green, red otherwise.

Behind the scenes, at each iteration the software computes the 3D mirror shape and generates the statistics for the objective function. Surface area, bounding box, and focal length constraint terms are immediately given by the resulting geometry. Sensor coverage is estimated by tracing 10,000 rays from the camera towards the mirror and counting missed intersections. We model the angular spread as the maximum angle at the scene center between any pair of virtual camera positions (the reflections of the real camera's optical center in the mirror facets), and express uniformity of the light field as the ratio between the maximum and minimum angles between neighboring virtual camera positions.

The observable scene extent can be expressed as follows: we consider a plane containing the center point of the scene and intersect the reflected rays of the camera's projection center to the edge midpoints of all mirror segments with this plane. The minimum distance of these intersection points to the scene center gives a radius of scene points that can be observed by all virtual cameras. We let the optimizer solve for the optimal orientation of the scene. As the optimizer does not take rays that miss the scene into account, it can artificially under-report the usable scene volume. However, for the parameter it finds, it guarantees that the reported scene volume can be completely observed.

The convergence speed varies with the number of active constraints and the error landscape; our implementation typically converges in less than one second on a commodity PC.

\section{Evaluating mirror shapes}

In order to show the flexibility provided by our interactive mirror designer, as well as the ease with which a user can find an appropriate design, we consider a number of case studies. The scenarios involve different imagers, different working volumes, and vastly different goals. Ray traced simulations of the resulting mirror shapes are shown in Figure 5.

Macro / narrow field of view: First, we consider a macro photography scenario for the capture of scenes $50 \mathrm{~mm}$ in diameter (see Figure 4 for a screenshot of the optimizer with all parameters and properties). We design the mirror for a full-frame DSLR and aim at a refocusing application. This requires the virtual camera positions to be close together, implying a small angular spread of 5 degrees. The resulting mirror is nearly planar, with rather shallow angles between facets. Constraints are almost all satisfied, the only blemish being angle ratio (at 1.4). This is the design we manufactured and used for Figure 1 and the results shown in Figure 8.

Macro / wide field of view: We modify the design to record the same scene type, but with a multiview stereo reconstruction application in mind (which requires a greater range of angles), increasing the angular spread to 35 degrees, while relaxing some of the manufacturing constraints. The optimization results in a design that leads to a highly uniform distribution of virtual cameras trading in some sensor coverage (92\% left) and a slightly higher visual distortion (Figure 5 , second from left) compared to the previous design.

Cell phone: The next scenario investigates a design that employs a small sensor typical for cell phone built-in cameras $(5.76 \times 4.29 \mathrm{~mm})$ for a scene diameter of $300 \mathrm{~mm}$. This results in comparatively large mirror $(454 \times 330 \times 50 \mathrm{~mm})$. 


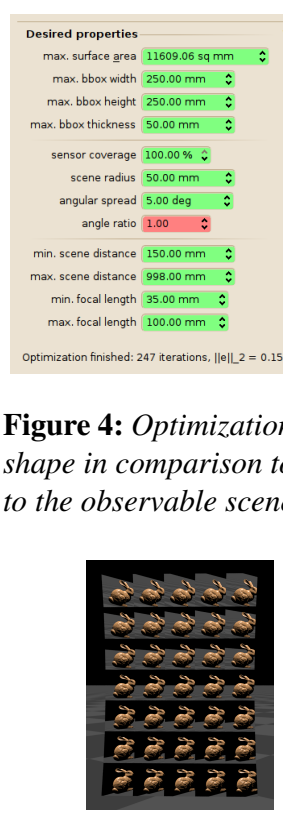

Macro, small fov
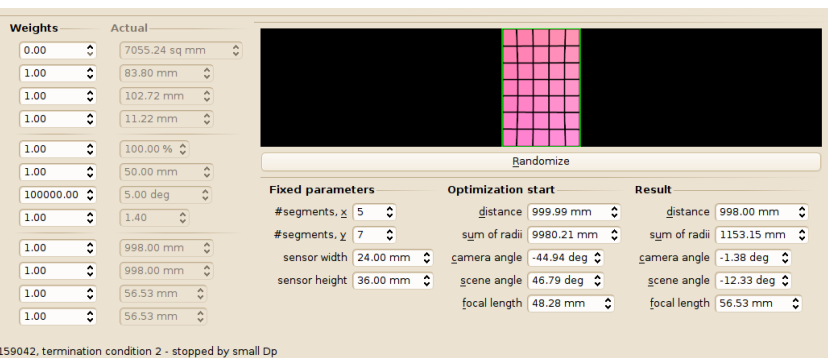
\#segments, $x=$ Fensor with $24.00 \mathrm{~mm}$ sensor width $24.00 \mathrm{~mm}$
sensor height $36.00 \mathrm{~mm}$
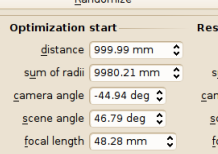
dstance $999.99 \mathrm{~mm}$ sum of radi $9980.21 \mathrm{~mm}$

nesult

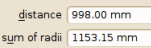

sum of radi $1153.15 \mathrm{~mm}$
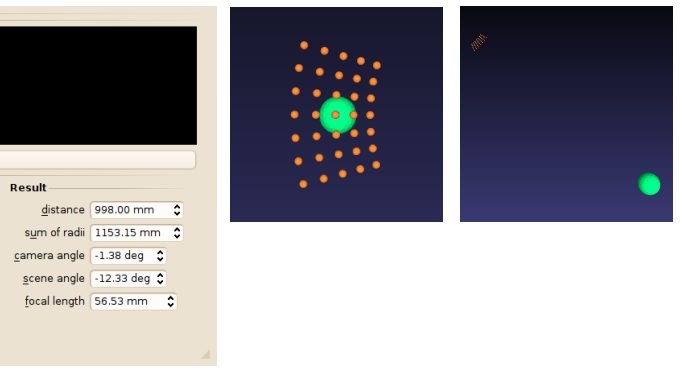

.

tant visualization of the mirror shape in comparison to the picture visible in the camera (green rectangle). Right: virtual camera locations (orange) compared to the observable scene center (green). The diameter of the green sphere corresponds to the diameter of the observable scene.

Figure 5: Ray tracings for the mirror designs used in the case study: the Stanford bunny is rendered floating atop a checkerboard as seen from the camera. The bunnies have been individually scaled to fill the field of view; their absolute size is comparable within the pairs of "macro" and "material measurement" examples.

In this case a "perfect" design is hard to achieve, requiring a compromise among several desirables (e.g., the resulting scene radius is $469 \mathrm{~mm}$ ). As the resolution of such a camera is lower than that of a DSLR, fewer segments must suffice.

Material measurement: Given the prior use of ellipsoidal mirrors for BRDF measurement, it is natural to use our interactive design program to find a construction that provides many views under a large set of angles. In contrast to mirrors with continuous curvature, our segmented design enables the observation of an extended scene region $(10 \mathrm{~mm}$ by $10 \mathrm{~mm})$ which enables the capture of a surface light field [WAA* ${ }^{*}$ ] of a small material patch; combined with controlled, changing illumination, this could be used to capture a spatially varying BRDF.

This design, which aligns the symmetry axis of the ellipsoid with the shorter image edge, provides the largest angular coverage among those we explored, at the cost of the greatest distortion (see Figure 5, fourth from the left) and greatest inefficiency with respect to usage of the sensor area $(79 \%)$. Note that the distortions on the right-hand side of Figure 5, third from the left) reduce the volume that can actually be observed by all facets considerably.

To amend the deficiencies of the above configuration, we also investigate a design which rotates the camera back to landscape orientation, aligning the larger image edge with the symmetry axis, and reduce the weight of the angular cov- erage penalty term. As could be expected, this results in a lower angular spread $\left(111^{\circ}\right)$, but also brings a considerable improvement with respect to distortions (see Figure 5).

\section{Manufacturing the mirror}

One of the major motivations for our automated mirror design pipeline was the widespread availability of computercontrolled rapid-manufacturing systems capable of high precision and low fabrication cost. The flexibility of these systems permits relatively few constraints in the design optimization process, allowing any final design to be turned into a physical prototype within a few hours. We have explored two manufacturing strategies, offering different tradeoffs between overall geometric accuracy and surface finish quality.

\subsection{Plated brass}

We used a milling machine under computer numerical control (CNC) to mill the surface geometry into a solid brass block (Figure 6). Because this process leaves residual grooves along the path the drilling tool took, machineassisted polishing is used to create planar surfaces. Bright nickel plating then creates a thin coating on the surface, providing a durable mirror finish (Figure 6). The mirror in the illustration was approximately $20 \times 13 \times 5 \mathrm{~cm}$, with $11 \times 7$ 

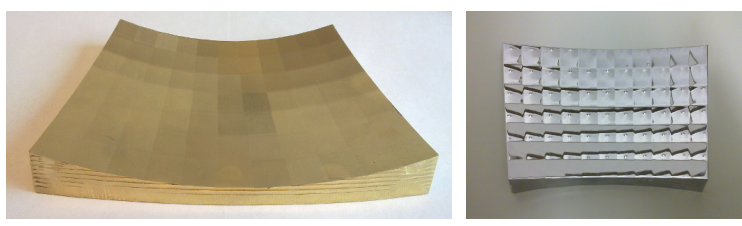

Figure 6: Mirror construction from electroplated brass. The mirror shape top is cut out from a solid brass block with a CNC mill. Polishing and electroplating with a bright nickel finish creates the desired mirror surface.

facets. Including the cost of raw materials, milling, polishing, and plating, the total build cost was on the order of USD 750

The clear advantages of this approach are its reliance on standardized rapid manufacturing processes to achieve a precise alignment. Indeed, we observe that the facets are oriented correctly, to a high degree of accuracy. In addition, manufacturing cost is essentially independent of the number of facets (depending almost entirely on the volume and area of the piece), leading to practical fabrication of mirrors with many dozens of facets.

A major drawback of this process, however, is that it is difficult to make the facets perfectly flat. Marks left by the milling tool remain visible even with a substantial amount of polishing. In addition, the polishing process smooths out the corners between facets, leading to an unusable area around the edges of each facet.

These tradeoffs are illustrated in Figure 7, which shows the reflection of a checkerboard target in the manufactured mirror described above. The precise alignment of the marked red checkerboard squares in the rectified images shows that the facets are pointed in the expected direction. However, some waviness is visible when zooming into the image, and there is substantial distortion (caused by corner smoothing) around the edges of each facet.

As in this design, the geometry of the mirror is very precisely known, it itself may be used to calibrate the parameters of the capturing camera by aligning a rendered version of the mirror geometry against the observed picture.

\subsection{Individual stainless steel facets}

An alternative manufacturing process is to separate the construction of the mirroring surface of each facet from its alignment. In this process (Figure 1) we mill a support structure from acrylic, then glue on individual facets that are cut from a sheet of mirror-polished stainless steel.

One major advantage of this design is lower manufacturing cost. The plating process, with its high setup cost, is avoided, and the support structure may be milled from inexpensive acrylic rather than brass. While both approaches scale with surface area, as the surface area needs to be milled out of the support structure, the milling process for the acrylic is faster and thus less expensive, as the acrylic is ablated more easily, and the surface can be cut with a smaller tool path density (residual grooves would be covered by the steel facets). Cutting the facets is more efficient, as the cutting time and cost scale with the length of the edges as opposed to surface area.

One disadvantage is that, after gluing the facets on the mirror support, their alignment is less precise than in the electroplated construction; we deal with that by having the user click on a single visible scene point in each segment and optimizing for individual rotation of the segments so that the corresponding rays converge at some point in space.

For cutting the facets, we investigated several technologies; CNC milling a $0.762 \mathrm{~mm}$ thick steel sheet induced distortions (Figure 7) due to mechanical stress. For a $1.524 \mathrm{~mm}$ thick sheet, they were reduced; we achieved the best results with laser-cut facets, as seen in Figure 1 and 7. They are mostly free of distortion, but the material used for them has suboptimal mirroring qualities, inducing a small loss of contrast which can be easily compensated by tonal curve edits in any photo processing program.

\section{Discussion}

Example recordings: We have experimented with several scenes recorded with the mirror design from Figure 1, which enables refocusing applications by means of projective texturing of a virtual plane, as demonstrated in Figure 8 and in the supplemental video. With only 35 input views, gaps can be seen; however, the sampling is sufficiently dense to permit effective view interpolation with optical flow [ZBW11]; after upsampling to 1617 views, most gaps are closed. As we use a single sensor with globally synchronized shutter for recording, we can acquire quickly changing scenes with short exposures, such as a splash of liquid against a spoon.

Comparison to existing systems: The design choices of the proposed catadioptric setup for light field recording set it apart from existing techniques as follows: in contrast to a moving camera, it enables single exposure recording. This is also possible with camera arrays, which have the additional benefit of being able to extend the number of views with almost linear cost by adding more cameras. As an advantage, though, our approach does not require synchronization, and, the mirror being a compact, single piece of equipment, it can be calibrated globally.

This is also the main difference to previous assemblies of mirror segments: as a consequence of the use of precise machining tools during the construction, the position of the mirror facets is either an intrinsic result of the construction process (in the plated brass version), or easy to do by hand (when aligning pre-cut mirror facets on acrylic support).

In addition, we make use of an integrated design pipeline, 

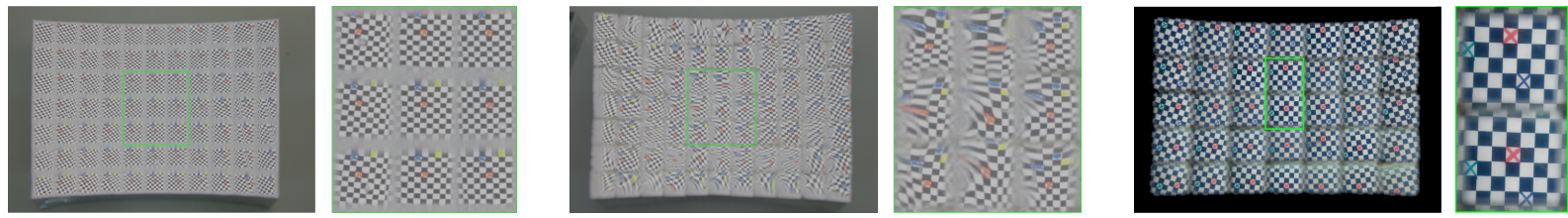

Figure 7: Comparison of manufacturing alternatives. Left: Electroplated brass mirror as seen by the camera and a magnification of the center region: several surface sections show blemishes which are reminders of the tool path that were not polished out, and artifacts introduced in the coating process. Center: Polished steel plates, manually cut out of a $0.762 \mathrm{~mm}$ thick steel sheet. This is a fast and inexpensive means of producing the mirror, but the distortions induced by mechanical stress during the cutting process render this particular configuration unusable. Right: precise laser cutting avoids these distortions.
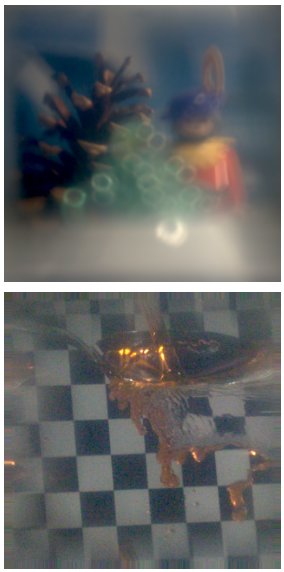
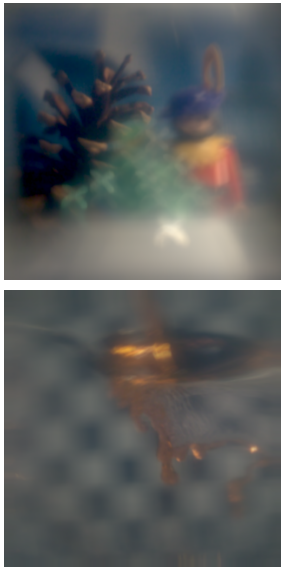
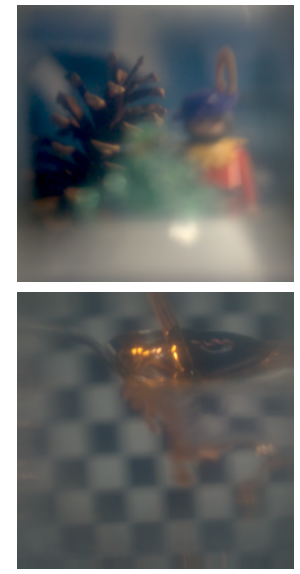
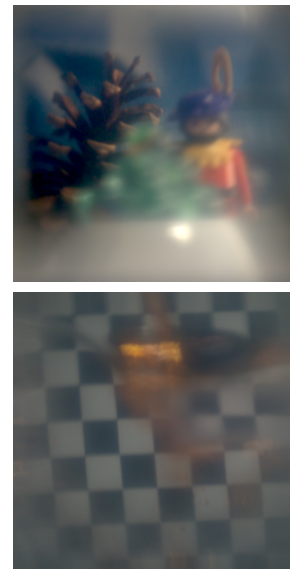
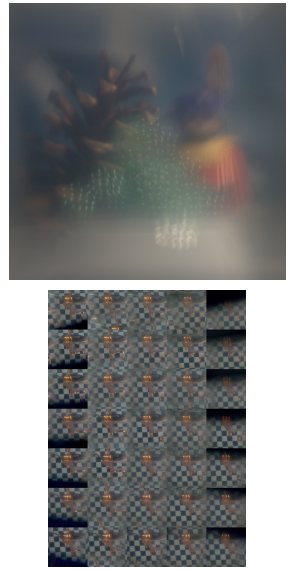

Figure 8: Refocusing results for light fields recorded with a faceted mirror setup after upsampling to 1617 images. The top row shows the result of simulating different aperture shapes for artistic bokeh control, the bottom row demonstrates refocusing for a short-exposure recording of liquid splashing off a spoon. The rightmost column shows refocusing using only the original 35 views (top) and the rectified input views (bottom). Please refer to the supplemental video for more examples.

which relieves the user from manually constructing mirror geometry, and supports the setup construction throughout from the definition of design goals over visualizations of mirror shapes and results up to the final construction.

Camera-internal modifications with refractive elements are more compact and, once assembled, easier to transport. They are however limited to applications that can make do with a small range of observed viewing angles such as refocusing as limited by the entrance pupil of the camera. In contrast, our method can provide solutions for much more diverse application scenarios.

Future work: Most beneficial extensions to the presented research will include broadening the space of mirror shapes that is considered in the optimization process. Extensions to non-planar facet geometry could do away with the minimal focal lengths in the virtual views, as convex facets would facilitate almost arbitrarily wide fields of view. With irregular mirror geometry, different sampling densities may be achievable which could make super-resolution possible.

The manufacturing process can be further improved to de- liver higher precision in the local surface geometry, and mass production techniques may enable the inexpensive duplication of the same mirror shape from a single mold.

In conclusion, we have presented a comprehensive method for mirror-based light field recording which leverages algorithmic control throughout the entire pipeline from design to construction on the path towards true post-hocphotography.

\section{Acknowledgements}

We thank Lawrence McIntyre for his support in the construction of the prototypes, Nicholas Johnson for fruitful discussion regarding the manufacturing processes, Yiming Liu for his help with some of the setup, Anette Müller for her support while recording the result pictures, Matt Pharr and Greg Humphreys for the PBRT software and Paolo Cignoni and colleagues for MeshLab [CC]. This work was supported by a fellowship within the Postdoc-Programme of the German Academic Exchange Service (DAAD) and US NSF grant \#CCF-1012147. 


\section{References}

[AVR10] Agrawal A., Veeraraghavan A., Raskar R.: Reinterpretable imager: Towards variable post capture space, angle \& time resolution in photography. Computer Graphics Forum (Proc. Eurographics special isssue) 29 (2010), 763-772.

[CC] Cignoni P., Colleauges: Meshlab. Visual Computing Lab -ISTI -CNR. URL: http: / / meshlab. sourceforge. net/.

[CNR08] COSSAIRT O., NAYAR S. K., RAMAMOORThi R.: Light field transfer: Global illumination between real and synthetic objects. ACM Trans. on Graphics (Proc. SIGGRAPH special issue) (Aug 2008).

[Dan01] DANA K. J.: BRDF/BTF measurement device. In ICCV Proceedings of Eighth IEEE International Conference on Computer Vision (Vancouver, British Columbia, July 2001), vol. 2, pp. 460-6.

[GGSC96] Gortler S. J., GRZESZCZUK R., SZELISKI R., CoHEN M. F.: The lumigraph. In Proc. SIGGRAPH '96 (New York, NY, USA, 1996), ACM, pp. 43-54.

[GHAO10] Ghosh A., Heidrich W., Achutha S., O'Toole M.: A basis illumination approach to BRDF measurement. International Journal of Computer Vision 90 (2010), 183-197. 10.1007/s11263-008-0151-7.

[GZC*06] Georgiev T., Zheng K. C., Curless B., SAlesin D., NAYAR S., INTWALA C.: Spatio-angular resolution tradeoffs in integral photography. In Rendering Techniques 2006 (Proc. Eurographics Symposium on Rendering) (2006), pp. 263272.

[IMG00] Isaksen A., McMillan L., Gortler S. J.: Dynamically reparameterized light fields. In Proc. SIGGRAPH 'OO (2000), ACM, pp. 297-306.

[IWH10] IhrKe I., WETZSTEIN G., HEIDRICH W.: A theory of plenoptic multiplexing. In Computer Vision and Pattern Recognition (CVPR), 2010 IEEE Conference on (2010), pp. $483-490$.

[LCV*04] Levoy M., Chen B., Vaish V., Horowitz M., MCDowall I., Bolas M.: Synthetic aperture confocal imaging. ACM Trans. Graph. (Proc. SIGGRAPH special issue) 23 (August 2004), 825-834.

[LCW*06] Lanman D., CRispell D., WaChs M., , TAUbin G.: Spherical catadioptric arrays: Construction, multi-view geometry, and calibration. In Third International Symposium on 3D Data Processing, Visualization and Transmission (2006).

[LH96] LEVOY M., HANRAHAN P.: Light field rendering. In Proc. SIGGRAPH '96 (New York, NY, USA, 1996), ACM Press, pp. $31-42$.

[LHG*09] Levin A., HasinofF S. W., Green P., Durand F., FreEmAN W. T.: 4D frequency analysis of computational cameras for depth of field extension. ACM Trans. Graph. (Proc. SIGGRAPH special issue) 28 (July 2009), 97:1-97:14.

[Lou04] LOURAKIS M.: levmar: Levenberg-marquardt nonlinear least squares algorithms in C/C++, July 2004. [Accessed on 15 Jan. 2011. Version used: 2.5]. URL: http://www. ics . forth.gr/ lourakis/levmar/.

[MP04] Matusik W., Pfister H.: 3D TV: a scalable system for real-time acquisition, transmission, and autostereoscopic display of dynamic scenes. ACM Transactions on Graphics (Proc. SIGGRAPH 2004) 23, 3 (2004), 814-824.

[MSY07] Mukaigawa Y., Sumino K., Yagi Y.: High-speed measurement of BRDF using an ellipsoidal mirror and a projector. In Procams: IEEE International Workshop on ProjectorCamera systems (2007).
[MTK*10] Mukaigawa Y., TAgawa S., Kim J., Raskar R. Matsushita Y., YAGI Y.: Hemispherical confocal imaging using turtleback reflector. In ACCV (1) (2010), Kimmel R., Klette R., Sugimoto A., (Eds.), vol. 6492, Springer, pp. 336-349.

[Ng06] NG R.: Digital light field photography. PhD thesis, Stanford University, 2006.

[NLB*05] NG R., LeVoy M., BRÉdif M., Duval G., HoROWITZ M., HANRAHAN P.: Light field photography with a hand-held plenoptic camera. Stanford University Computer Scienece Tech Report (April 2005)

[PHD06] PeERs P., HaWkins T., DebeVEC P.: A reflective light stage. ICT Technical Report ICT-TR-04.2006 (2006).

[TARV10] TAguchi Y., AgraWal A., Ramalingam S., VEERARAgHAVAN A.: Axial light field for curved mirrors: Reflect your perspective, widen your view. In Computer Vision and Pattern Recognition (CVPR), 2010 IEEE Conference on (2010), pp. $499-506$.

[TAV*10] Taguchi Y., Agrawal A., Veeraraghavan A., RAMALINGAM S., RASKAR R.: Axial-cones: Modeling spherical catadioptric cameras for wide-angle light field rendering. ACM Transactions on Graphics (Proc. of SIGGRAPH Asia special issue) (2010).

[UWH*03] Unger J., Wenger A., HaWkins T., Gardner A., DeBEVEC P.: Capturing and rendering with incident light fields. In Rendering Techniques 2003 (Proc. Eurographics Symposium on Rendering) (June 2003), pp. 141-149.

[VAR*08] Veeraraghavan A., Agrawal A., Raskar R., MOHAN A., TUMBLIN J.: Non-refractive modulators for encoding and capturing scene appearance and depth. In Computer Vision and Pattern Recognition, 2008. CVPR 2008. IEEE Conference on (2008), pp. 1 -8.

[VRA*07] Veeraraghavan A., Raskar R., Agrawal A., Mohan A., TUMblin J.: Dappled photography: mask enhanced cameras for heterodyned light fields and coded aperture refocusing. ACM Transactions on Graphics (Proc. SIGGRAPH 2007) 26,3 (2007), 69.

[WAA*00] Wood D. N., Azuma D. I., Aldinger K., CurLess B., Duchamp T., SAlesin D. H., Stuetzle W.: Surface light fields for 3D photography. In Proc. SIGGRAPH 'OO (New York, NY, USA, 2000), ACM Press/Addison-Wesley Publishing Co., pp. 287-296.

[War92] WARD G. J.: Measuring and modeling anisotropic reflection. In Proc. SIGGRAPH '92 (1992), pp. 265-272.

[WIH10] WetzSTEIN G., IHRKe I., HeIDRICH W.: Sensor saturation in fourier multiplexed imaging. In Computer Vision and Pattern Recognition (CVPR), 2010 IEEE Conference on (2010), pp. $545-552$.

[WJV*05] Wilburn B., Joshi N., Vaish V., Talvala E.-V., Antunez E., BARth A., AdAms A., Horowitz M., LeVoy M.: High performance imaging using large camera arrays. ACM Trans. Graph. 24 (July 2005), 765-776.

[Yan00] YAng J. C.: A Light Field Camera For Image Based Rendering. PhD thesis, Massachusetts Institute of Technology, 2000.

[YeBM02] Yang J. C., Everett M., Buehler C., McMilLAN L.: A real-time distributed light field camera. Rendering Techniques 2002 (Proc. Eurographics Rendering Workshop) (2002), 77-86.

[ZBW11] ZimMER H., BRUHN A., Weickert J.: Optic flow in harmony. International Journal of Computer Vision 93, 3 (2011), 368-388. 